\title{
Different Interaction Mechanisms of Evaporated Porphyrin Films Exposed to $\mathrm{NO}_{2}$
}

\author{
M. Rivera1', J. M. Rivera1, 0. Amelines-Sarria², Y. A. Wang3 \\ ${ }^{1}$ Instituto de Física, Universidad Nacional Autónoma de México, Mexico City, Mexico \\ ${ }^{2}$ Universidad Pontificia Bolivariana, Bucaramanga, Colombia \\ ${ }^{3}$ University of British Columbia, Vancouver, Canada \\ Email: mrivera@fisica.unam.mx, oscar.amelines@upb.edu.co,ya wang@chem.ubc.ca
}

How to cite this paper: Rivera, M., Rivera, J.M., Amelines-Sarria, O. and Wang, Y.A. (2018) Different Interaction Mechanisms of Evaporated Porphyrin Films Exposed to $\mathrm{NO}_{2}$. Advances in Materials Physics and Chemistry, 8, 441-457.

https://doi.org/10.4236/ampc.2018.811030

Received: September 3, 2018

Accepted: November 13, 2018

Published: November 16, 2018

Copyright (C) 2018 by authors and Scientific Research Publishing Inc. This work is licensed under the Creative Commons Attribution International License (CC BY 4.0).

http://creativecommons.org/licenses/by/4.0/

\begin{abstract}
In this work, metal free and zinc tetraphenylporphyrin films were employed as nitrogen dioxide $\left(\mathrm{NO}_{2}\right)$ gas sensors. The films were vacuum evaporated and the sensor response was evaluated as changes in the optical absorption spectra, hydrophobic properties and conductivity at different gas concentrations. From UV-Vis results, important changes in the absorption peaks were observed after gas exposure. The morphology of the films before and after gas interaction was obtained by using scanning electron and atomic force microscopy. The films morphology showed a degradation after gas adsorption for the metal free system but gas entrapment for the zinc porphyrin film. In order to elucidate the gas adsorption phenomena, density functional theory calculations were also performed. Here, it was observed that the porphyrin chemical structure not only affects the gas coordination sites which affect the porphyrin electronic distribution and packing arrangement, but also, determines the gas detection mechanism for sensing applications.
\end{abstract}

\section{Keywords}

Porphyrin Films, Vacuum Evaporation, $\mathrm{NO}_{2}$, Gas Sensor,

Density Functional Theory

\section{Introduction}

Nowadays, most of the current commercially available detectors of volatile organic compounds (VOC) have been fabricated by using metal oxide semiconductors, and they employ detection techniques such as photoionization, electrochemistry and non-dispersive infrared and thermal methods, which require several detection steps, expensive materials and complex signal generators [1]. For 
these reasons, people have started using more environmental friendly compounds and less complex techniques for chemical detection and recognition of harmful volatile toxic compounds in the environment. Recently, more work has been devoted to investigate the role of organic films as promising detector materials. In particular, conjugated macrocycles such as phthalocyanines and porphyrins are interesting molecules since they exhibit rich redox activity, a well defined characteristic absorption spectra and the ability to transfer charge carriers, and therefore, interesting conductive properties, among others. For instance, changes in the color and the electrical conductivity of these films after specific gas interactions have suggested that these systems represent a reliable and economical method to produce portable gas sensor devices [2].

While phthalocyanines have been most commonly employed as chemical sensors [3] [4], less work has been devoted to use porphyrin and metalporphyrin films in spite that this molecule plays a key role as active organic material for various biological processes [5]. Over the last few years, some experiments have been carried out to investigate the sensitivity of porphyrins in solution [6], thin films formed with the Langmuir-Blodgett technique [7] [8] [9] [10] and spin-coating [11] to detect volatile compounds. On the other hand, Roales et al. [12] showed the influence of the porphyrin central metal group in the sensing response of volatile organic compounds. In that study, the zinc porphyrin film exhibited by far the most important changes among different central metal atoms. Furthermore, metal and metal free porphyrin films have been employed to detect $\mathrm{CO}_{2}$ and $\mathrm{NO}_{2}$ toxic gases, among others [8] [9] [10].

Because of their distinctive bright colors, porphyrin species exhibit very distinct absorption bands in the visible spectrum due to electronic transitions between HOMO and LUMO states [13]. The highly conjugated electronic structure of the porphyrin ring gets modified by the strong interaction with the volatile gases resulting in changes in the porphyrin absorbance bands [6].

In addition, the resulting conductivity of these films during gas exposure, not only depends on the molecular structure (metallic center and functional groups), but also on the analyte specie. Oxidizing gases such as $\mathrm{NO}_{2}$ have attracted considerable attention since they can be found in the atmosphere, and are considered highly toxic with very harmful effects for all life forms. As pollutant, $\mathrm{NO}_{2}$ intervene in the conversion of different $\mathrm{NO}_{\mathrm{x}}$ species in the atmosphere, reacting with sunlight to produce ozone. Moreover, $\mathrm{NO}_{\mathrm{x}}$ species are involved in many biological processes and biomimetic catalytic reactions which are important at the cellular level [14].

Although previous works suggested that the sensing characteristics of porphyrin films remain active over long periods with short recovery times, little evidence has been given on the effects of the gas to the porphyrin films. Furthermore, some speculation has been done regarding the binding of the analytes to the molecules, but so far, little work has been done to determine the exact location and nature of this binding. Therefore, time-dependent density functional 
theory (TD-DFT) has been employed to investigate the fundamental electronic and optical properties of porphyrin molecules exposed to $\mathrm{NO}_{2}$. Previous calculations on Iron(II) porphyrin systems indicated that porphyrins mediate the reduction of nitrate into nitric oxide through two different pathways involving the $\mathrm{N}$-nitro and O-nitro-Fe(II) porphyrin complexes [15] [16]. It is worth noticing that metal free and zinc porphyrin systems have not been studied by using similar analysis.

Finally, gas sensors can be extremely sensitive to the environment, and therefore, small changes on the film interface could produce changes in the surface energy, and therefore, the sensing response. For this reason, contact angle measurements were carried out before and after gas exposure, in order to investigate changes in the hydrophobic properties of the films.

So, in this work, we employed metal-free meso-tetraphenylporhyrin and zinctetraphenylporphyrin films to investigate their capacity to detect $\mathrm{NO}_{2}$ by studying changes in the adsorption spectra, morphology, contact angle and the in-situ conductivity along the films. Since most of the work related to the detection of volatile compounds has been devoted to the detection limits by using thin porphyrin films [17] [18], in this work, we employed not only molecules with two different coordination centers but also thicker films in order to investigate possible differences in the sensing mechanisms. In addition, DFT Computer simulations were also performed in order to understand the experimental results, and provide some insights regarding the adsorption/absorption mechanisms for gas detection.

\section{Experimental and Theoretical Methods}

5, 10, 15, 20-tetraphenylporphyrin $\left(\mathrm{H}_{2} \mathrm{TPP}\right)$ and Zinc-5, 10, 15, 20-tetraphenylporphyrin (ZnTPP) compounds were purchased from Sigma-Aldrich (97\%) and used without further purification. The films were deposited with the physical vapour deposition technique onto quartz and indium tin oxide (ITO) substrates for the optical and electrical characterizations, respectively, by using the VCM600 system from Norm Electronics Ltd working at $10^{-6} \mathrm{mbar}$. Spectroscopic measurements of the films were carried out by a UV-Vis Blue-wave miniature StellarNet spectrometer before and after gas absorption in the $400-1000 \mathrm{~nm}$ wave range. The morphology of the films was investigated with a field emission scanning electron microscope JSM-7800F from JEOL, working at $2 \mathrm{kV}$ with the gentle beam mode in order to avoid damage to the films. Furthermore, atomic force microscopy images of the films interface were acquired with a JEOL JSPM-4210 microscope in the tapping mode. Both characterizations were performed before and after gas exposure to investigate morphological changes. The presence of the gas was also established with the energy dispersive spectroscopy technique by using elemental analysis with an Aztec energy standard microanalysis system. Conductive measurements of the films were acquired in a homemade device inside a gas chamber in order to investigate the in-situ conductivity 
of the films during was exposure. For this purpose, a dual tip setup connected to a power supply (Keithley 2400 SourceMeter) was employed. The conductive tips were placed in contact with the film surface at a constant DC current of $1 \mathrm{~mA}$, and the voltage variation during gas exposure was obtained as a function of time. The $\mathrm{NO}_{2}$ gas was produced by the chemical reaction of a high purity (99.9\%) copper wire and regent grade concentrated nitric acid 70\% from Sigma-Aldrich to obtain different gas concentrations ( $20 \mathrm{ppm}, 40 \mathrm{ppm}$ and $80 \mathrm{ppm}$ ) assuming a $100 \%$ conversion. The reactor container was connected to the $0.637 \mathrm{~L}$ conductive measurement chamber. The gas was introduced at a constant flow rate to maintain the same experimental conditions in all cases. All experiments were carried out at room temperature.

On the other hand, density functional theory (DFT) calculations were performed in order to investigate the molecular interactions between porphyrin and gas molecules by using the hybrid B3LYP/6-31(d,p) basis set, since it has provided good agreement with experimental results. For this study, all molecular geometries were fully optimized with the Gaussian 09 program package. For the simulations, two different configurations for each porphyrin system were considered: a) the interaction of one nitrogen dioxide group at different positions on the porphyrin molecules, i.e. $\mathrm{NO}_{2}$-porphyrin, and b) the interaction of two porphyrin molecules and one nitrogen dioxide group, i.e. porphyrin- $\mathrm{NO}_{2}$-porphyrin. In the later case, the porphyrin self-assembled structure was employed to provide not only relevant information regarding the possible packing of the molecules within the film, but also the interaction of the $\mathrm{NO}_{2}$ molecule within the stacked porphyrin layers.

\section{Results}

\subsection{Experimental Results}

Figure 1 and Figure 2 show the scanning electron microscopy images of the metal free and zinc porhyrin films before and after gas adsorption, respectively. Figures a), exhibit the ITO surface prior to the film deposition. Figures b), show the evaporated porphyrin films, which exhibited a very uniform and homogeneous deposit in both cases. Figures (c) and (d) show the surface after being exposed to $\mathrm{NO}_{2}$ at concentrations of $40 \mathrm{ppm}$ and $80 \mathrm{ppm}$, respectively. From these images, an important surface degradation can be seen in Figure 1. The $40 \mathrm{ppm}$ image shows branched-like defects covering over $80 \%$ of the surface. The image also show nucleation centers of different dimensions scattered along the surface. Higher magnification images of the interface (not shown here), exhibit defects with linear edges suggesting an ordered arrangement of the molecules within the film. An interesting aspect of the images is that underneath the defects, a porphyrin film can still be seen, which suggests that the gas interaction begins at the film interface. At $80 \mathrm{ppm}$ exposure, the extent of surface damage increased and became more homogeneous.

In Figure 2, the $40 \mathrm{ppm}$ image shows a homogeneous film with granular features randomly distributed and diameters between $0.5 \mathrm{um}$ to $1.0 \mathrm{um}$. At 80 

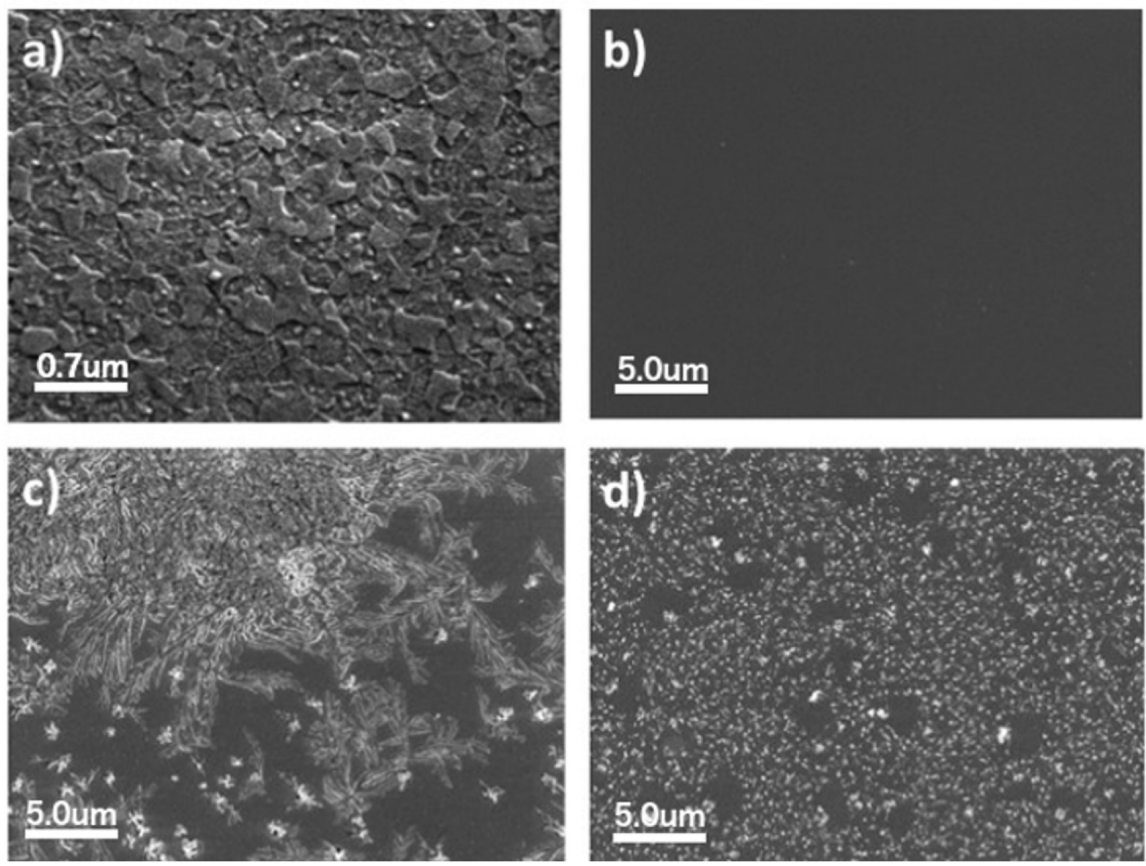

Figure 1. Scanning electron microscopy images of the $\mathrm{H}_{2}$ TPP film; (a) Bare ITO, (b) As-deposited, (c) after 40 ppm,(d) after 80 ppm gas exposure.
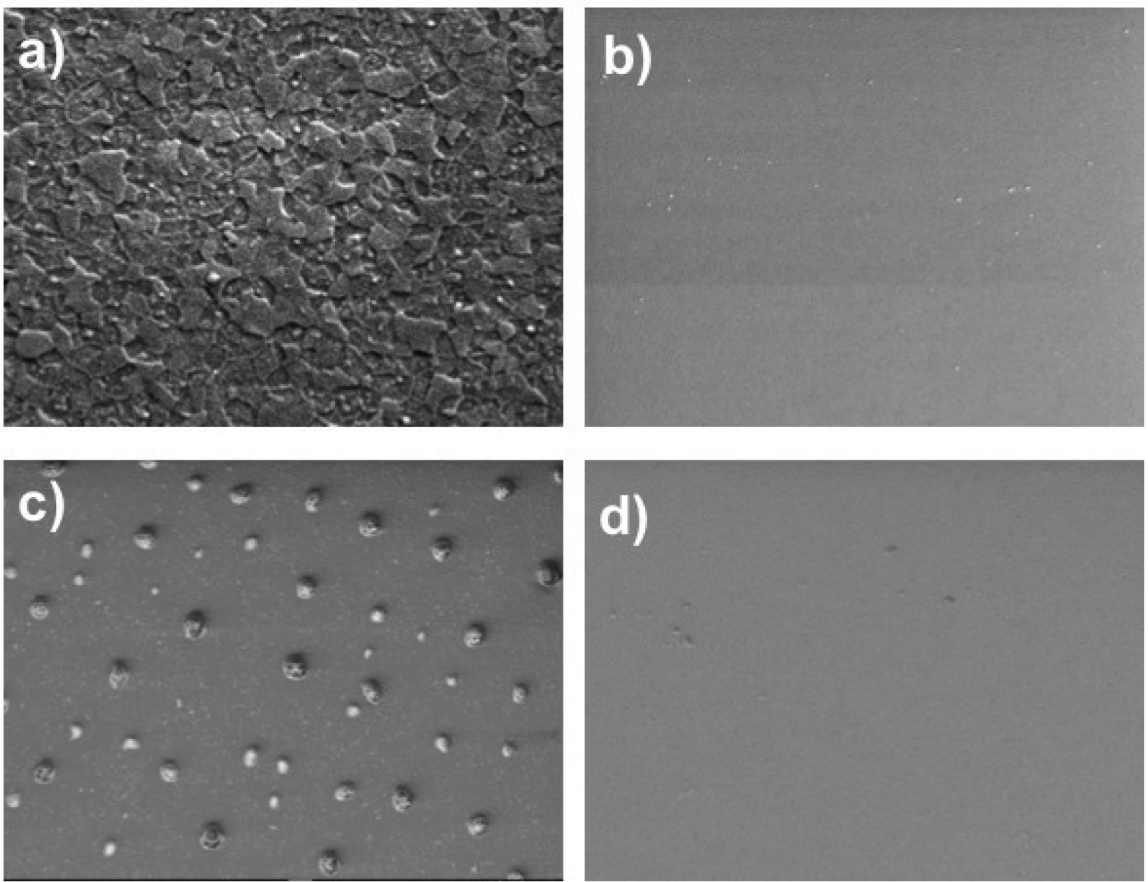

Figure 2. Scanning electron microscopy images of the ZnTPP film; (a) Bare ITO, (b) As-deposited, (c) after 40 ppm, (d) after 80 ppm gas exposure.

ppm exposure, the surface became more homogeneous but at higher magnification (not shown here), a more regular granular interface can be seen. In addition, the texture of these films after gas exposure is the same as the original interface, so, we can infer that these features do not change the original characte- 
ristics of the initial film. This result is different from the previous image (sem 1), which suggests that a different gas-film interaction mechanism took place.

In Figure 3 and Figure 4, atomic force microscopy images of the metal free and zinc porphyrin films before and after gas absorption are shown. Images a) and b) show the ITO surface and the porphyrin films after deposition, respectively. Then, after gas exposure, the surfaces show important differences attributed to distinct interaction phenomena between the films and the gas in agreement with SEM results. For the metal free film, as the gas concentration increased from $40 \mathrm{ppm}$ to $80 \mathrm{ppm}$, Figure 3(c) and Figure 3(d), show a progressive deterioration of the film suggesting that the degradation initiates at the exposed interface. The average thickness of the initial film was $160 \mathrm{~nm}$ and the roughness values of images in Figure 3 are: (a) $0.41 \mathrm{~nm}$, (b) $1.07 \mathrm{~nm}$, (c) 28.11 $\mathrm{nm}$ and (d) $23.79 \mathrm{~nm}$, respectively.

On the other hand, for the film containing the porphyrin with the zinc central metal atom, the surface aspect is completely different after gas exposure (Figure 4(c) and Figure 4(d)). Here, the texture at the interface is the same, although we can notice some depressions and regions of swelling represented by differences in color contrast. At $40 \mathrm{ppm}$, swelling areas exhibited semicircular shapes with maximum diameters of 3 um, approximately. Since the texture of the voids and swelling regions is the same, we infer that some gas got trapped underneath the film interface. Finally, the film after the $80 \mathrm{ppm}$ gas exposure showed a granular aspect with features up to $1 \mathrm{um}$ at the interface. In addition, some irregular depressions at the interface can be noticed, although the texture is the same for all the surface. The roughness values for these images are (a) $0.41 \mathrm{~nm}$, (b) $0.61 \mathrm{~nm}$, (c) $3.37 \mathrm{~nm}$ and (d) $11.93 \mathrm{~nm}$, respectively, and the original film thickness was $140 \mathrm{~nm}$.

The optical absorption spectra of the films before and after $\mathrm{NO}_{2}$ gas exposure for the a) metal-free and b) zinc porphyrine films are shown in Figure 5. Here, the as-deposited metal-free film spectra show the characteristic Soret $(433 \mathrm{~nm})$ and four Q bands (520, 553, 590 and $646 \mathrm{~nm}$ ) as seen in Figure 5(a). The broadened of the peaks is related to the molecular aggregation in the solid state. After gas adsorption, the Soret peak exhibits a bathochromic shift for all gas concentrations to $458 \mathrm{~nm}$. In addition, the $\mathrm{Q}$ bands disappear and one smooth shoulder and one distinct broad peak appear at $603 \mathrm{~nm}$ and $680 \mathrm{~nm}$, respectively. This spectrum is very similar to the one obtained after Farias et al. [8], although small differences can be attributed to the thickness and preparation methods of the films.

The spectra of the ZnTPP film, Figure 5(b), showed the Soret peak at $450 \mathrm{~nm}$ and two small Q peaks at $550 \mathrm{~nm}$ and $600 \mathrm{~nm}$, respectively, typical of metalloporphyrins in the $500-600 \mathrm{~nm}$ wavelength range [19]. After $\mathrm{NO}_{2}$ gas exposure, a large redshift of the Soret band to $521 \mathrm{~nm}$ occurred. In addition, the original Q bands disappeared and one smooth shoulder at $603 \mathrm{~nm}$ in addition to two peaks at $670 \mathrm{~nm}$ and $780 \mathrm{~nm}$ appeared. One interesting aspect of this result is related to the intensity of the peak at different gas concentrations. For instance, the 

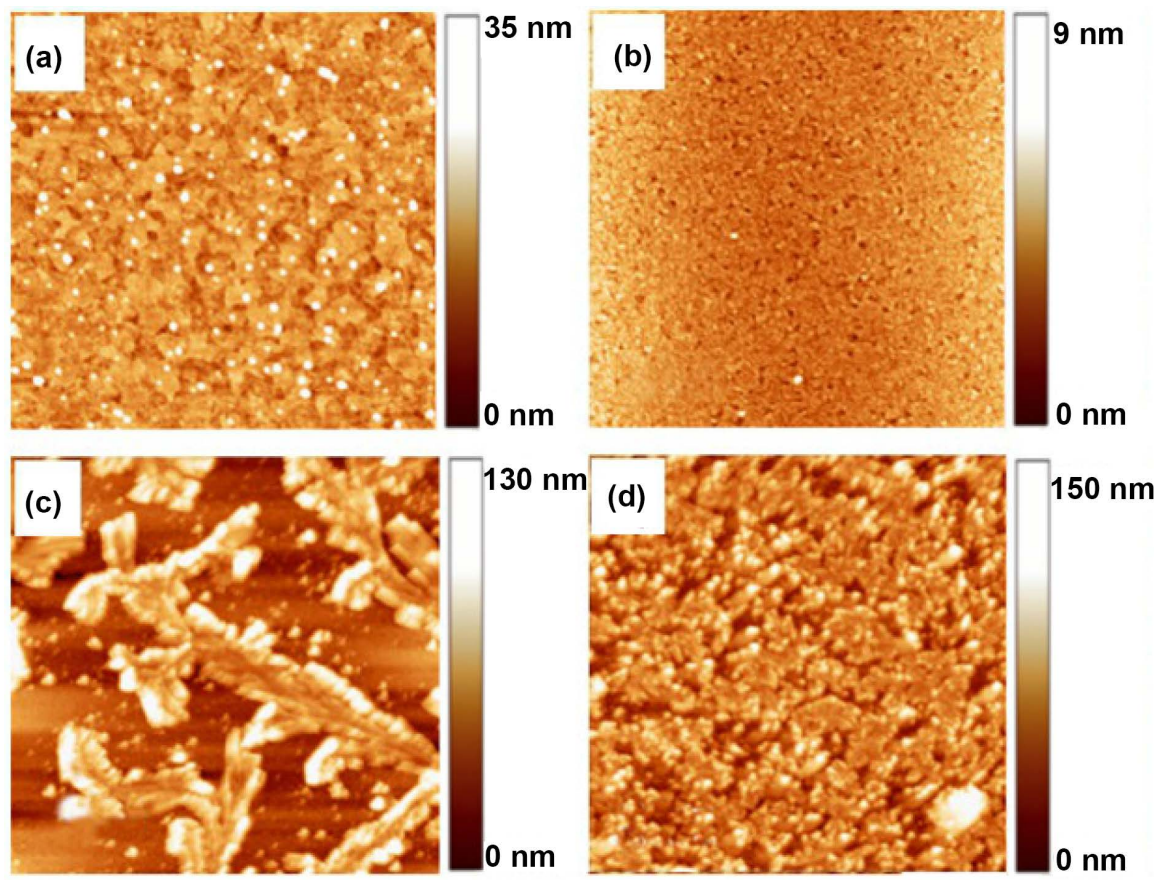

Figure 3. AFM images of (a) Bare ITO, (b) As-deposited $\mathrm{H}_{2}$ TPP film, (c) after 40 ppm gas exposure and $(\mathrm{d})$ after $80 \mathrm{ppm}$ gas exposure. Images are $10 \mathrm{um} \times 10 \mathrm{um}$
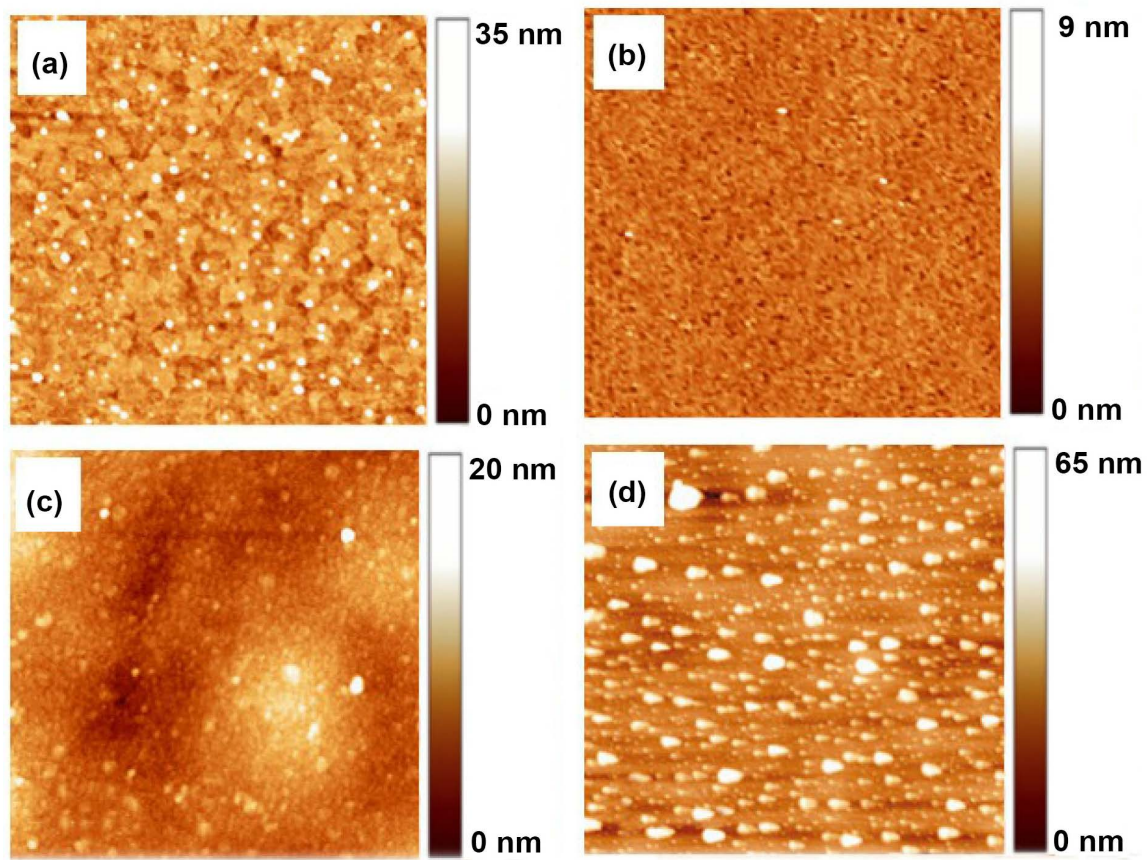

Figure 4. AFM images of (a) Bare ITO, (b) As-deposited ZnTPP film, (c) after 40 ppm gas exposure and (c) after $80 \mathrm{ppm}$ gas exposure. Images are $10 \mathrm{um} \times 10 \mathrm{um}$

Soret peak decreased its intensity as the gas concentration increased, while the new peaks between $650-800 \mathrm{~nm}$ showed the opposite trend. Another important feature of these plots is that the position of the new Soret and other peaks remained at nearly the same wavelength regardless of the gas concentration value. 


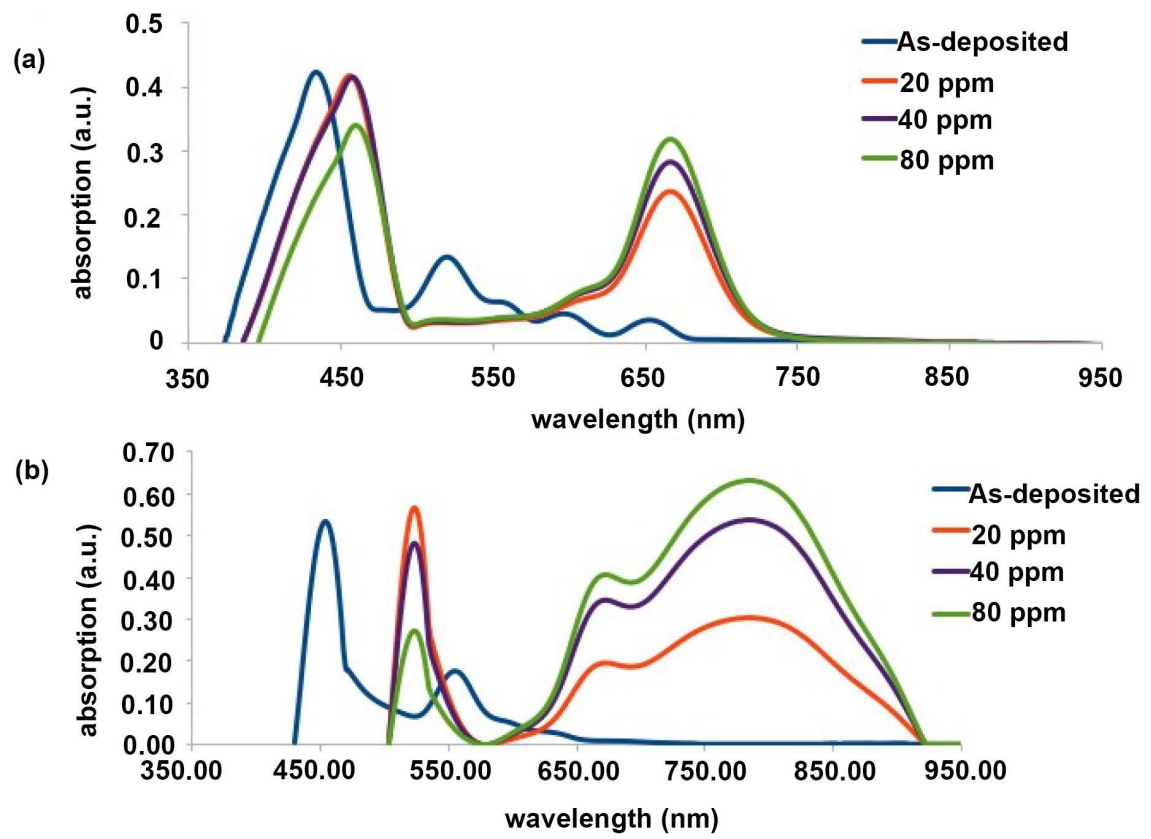

Figure 5. UV-Vis spectra of films before and after gas exposure for the (a) metal free porphyrin film and (b) zinc porphyrine film.

Some authors have suggested that the red shift is due to changes in the aggregation state of the molecules within the film [8], while others, that the shift is due to changes in the electronic structure of the porphyrin related to the electron withdrawing characteristic of the $\mathrm{NO}_{2}$ group [20]. In the metal porphyrin systems, it has been suggested that the new position of the Soret peak can be attributed to the binging of the gas to the central metal atom [21]. In order to investigate the nature of the peaks, UV-Vis was also performed for both porphyrins in chloroform at the same volume and gas concentrations. Here, we observed that the number and position of the peaks was fairly the same after gas exposure with respect to the porphyrin films, although the peaks were thinner and much better defined as expected. In addition, we did not observed changes in the absorption values for all gas concentrations. Since the porphyrin volumes were the same in all cases, the number of available porphyrin molecules for interaction was the same, and therefore, the intensity of the absorption peaks did not change as the gas concentration increased. The results in the solid state are different since the main interaction of the gas is initially with the molecules at the film surface. In this case, the gas interaction begins at the interface, and as the gas molecules reach the molecular film, the number of porphyrin molecules interacting with $\mathrm{NO}_{2}$ augments as the gas concentration increases (either by adsorption or absorption, depending on the porphyrin molecular structure), which corresponds to the observed UV-Vis absorption plots. In the next section, we will provide more information regarding the origin of the peaks after gas exposure.

The in-situ and in-plane conductivity response of the metal-free and zinc porphyrin films for the various $\mathrm{NO}_{2}$ concentrations is shown in Figure 6 . From these plots, it is clear that the voltage conductivity increases as the gas 


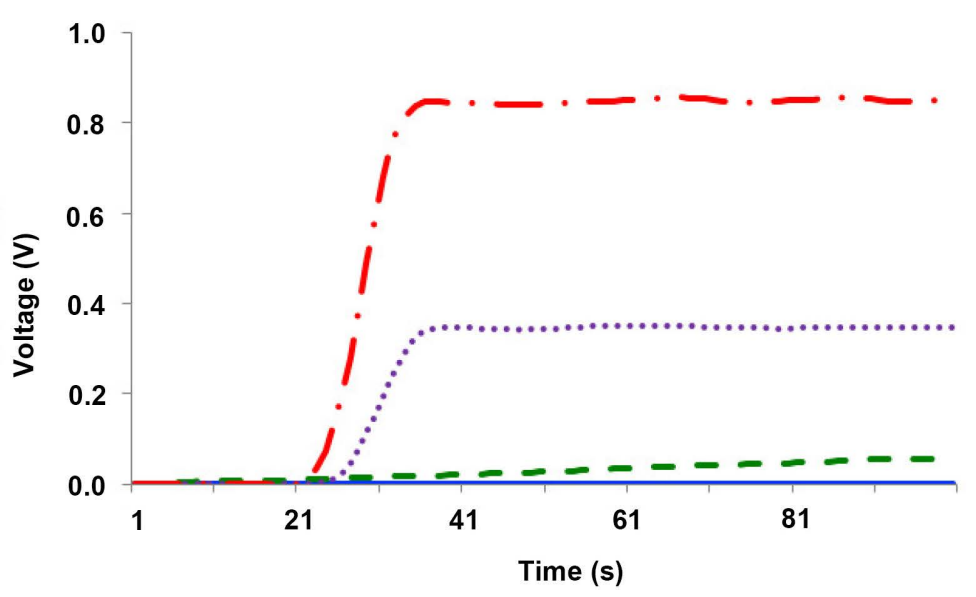

- No Gas

- $20 \mathrm{ppm}$

‥40 ppm

-80 ppm

(a)

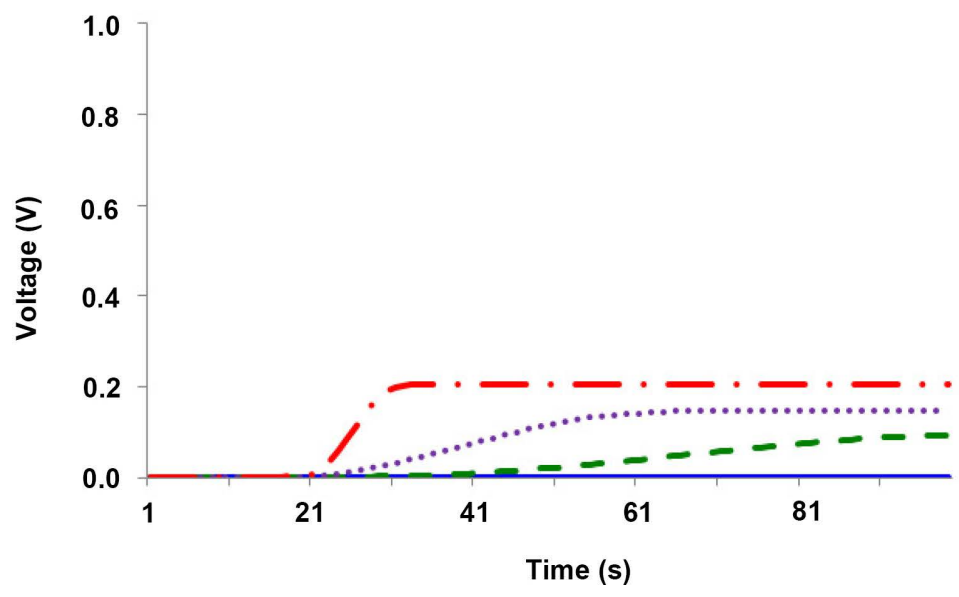

- No Gas

- 20 ppm

..440 ppm

-80 ppm

(b)

Figure 6. In-plane electrical conductivity measurements of films before and after gas exposure. a) Metal free porphyrin and b) zinc porphyrin films.

concentration augments in both cases. As reference, the non-gas curve was included which showed a null response over time, as expected. The most important aspect of these plots is the magnitude of the saturation voltage between the two films. The conductive values are larger in the metal free system for all gas concentrations. In addition, the voltage response is faster in the former case as well as the saturation voltage. Similar results have been reported by Richardson et al. when using Langmuir-Blodgett metal-free porphyrin films [10]. On the other hand, previous reports of the in-plane conductivity of $\mathrm{Cu}$ (II) porpohyrin films deposited by the LB technique, showed that as the thickness of the film increased, the conductivity increased too, but it decreased over time as irreversible changes on the film took place [22].

Finally, contact angle measurements were performed before and after gas exposure $(80 \mathrm{ppm})$ for both films in order to investigate changes in the surface properties. In Figure 7, the plots of the contact angle for a) the metal free and b) Zinc porphyrin system are shown. Before gas exposure, the metal porphyrin film exhibited a more hydrophobic nature in contrast with the metal free film. After 


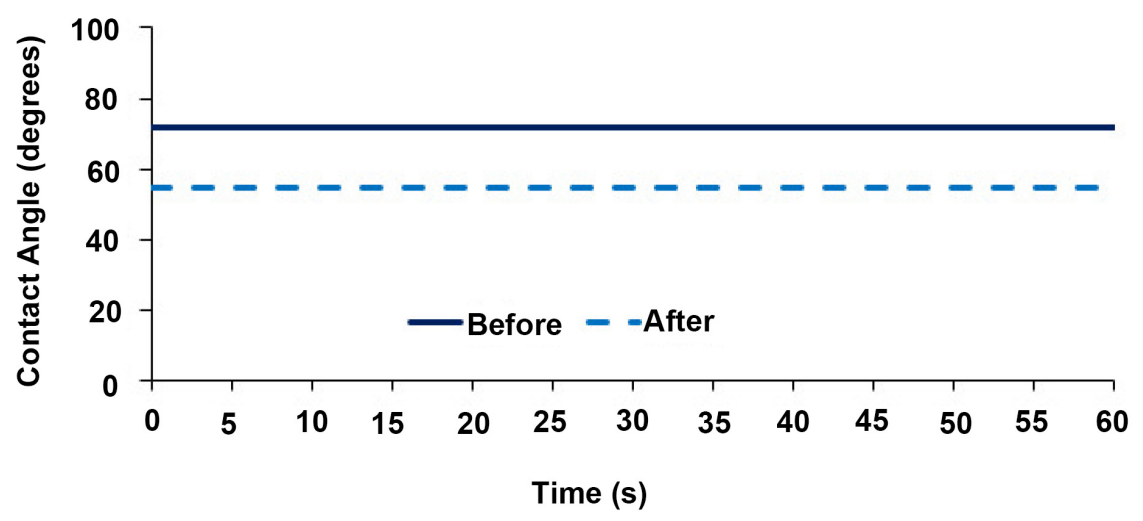

(a)

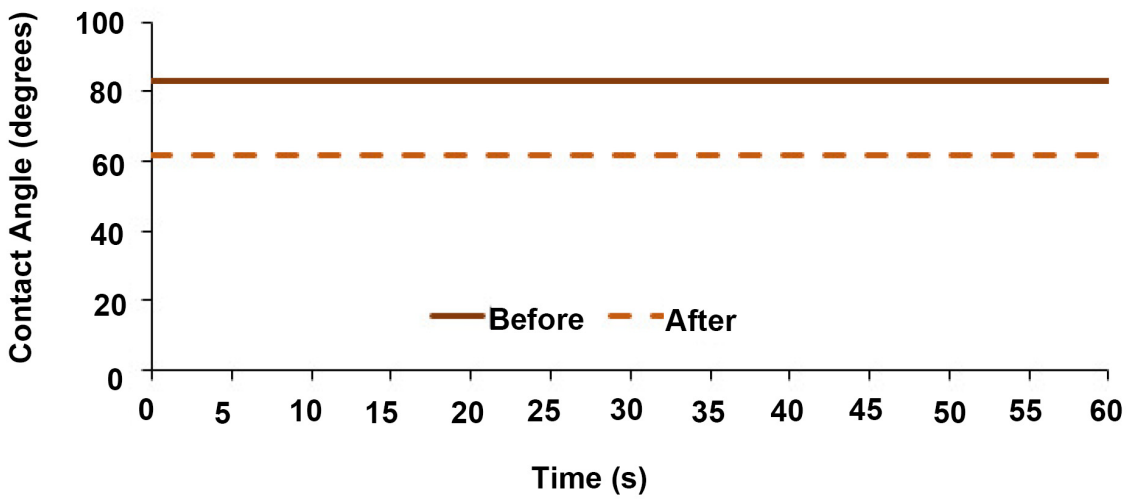

(b)

Figure 7. Contact angle measurements of films before and after $80 \mathrm{ppm}$ gas exposure. (a) metal free porphyrin and (b) zinc porphyrin films.

gas exposure, the contact angle decreased by 17 degrees for the metal free and 21 degrees for the metal film. Although the metal free film showed the lowest contact angle change, it also exhibited the highest surface roughness. Since both initial angles were lower than $90^{\circ}$, it was expected a larger decrease of the contact angle for the metal free film due to the roughness increase, but the angle value was lower in this case. Since both, the chemical and morphologic properties affect the wettability of surfaces, from the roughness results, we assume that the chemical transformation rather than the roughness has a larger influence on the hydrophobic behavior of the new surface.

\subsection{DFT Results}

The optimized structures of the $\mathrm{H}_{2}$ TPP, ZnTPP and gas molecules were used as starting configuration for the gas interaction. In both cases, the porphyrin ring exhibited a flat plane and the peripheral phenyl substituents were tilted $66.33^{\circ}$ with respect to the average plane of the macrocycles, in agreement with other works [23].

By simulating the interaction between the free metal porphyrin molecule and one $\mathrm{NO}_{2}$ group, the most stable configuration was found with the $\mathrm{NO}_{2}$ molecule 
$2.8 \AA$ above the porphyrin ring. The nitrogen atom pointed down towards the macrocycle, and it was located nearly above one of the inner nitrogen groups ( $7.67^{\circ}$ from the perpendicular direction towards the macrocycle plane). The oxygen atoms lied $0.54 \AA$ above the nitrogen plane at an angle of $7.67^{\circ}$ pointing away from the macrocycle center towards the $\alpha$ position in the macrocycle according to the fisher nomenclature. The internal $\angle \mathrm{O}-\mathrm{N}-\mathrm{O}$ angle was $132.05^{\circ}$ as seen in Figure 8. This configuration exhibited a binding energy of -12.27 $\mathrm{kJ} / \mathrm{mol}$.

The $\mathrm{ZnTPP}-\mathrm{NO}_{2}$ system, shows a planar ring where the most stable geometry corresponded to the coordination between one oxygen atom and the zinc metallic center in a cis-O-nitrito configuration, similar to other metal porphyrin systems interacting with $\mathrm{NO}_{2}$ groups [15] [21]. The binding energy of this arrangement was $-55.37 \mathrm{~kJ} / \mathrm{mol}$. The bond lengths $\mathrm{Zn}-\mathrm{O}$ and O-N were $2.06 \AA$ and $1.32 \AA$, respectively, and the bond angle $\angle \mathrm{O}-\mathrm{N}-\mathrm{O}$ was $117.99^{\circ}$. The vertical plane of the $\mathrm{NO}_{2}$ molecule aligned towards a meso-carbon position away to the central metal atom as seen in Figure 8(c)) and Figure 8(d)). One important aspect is that the $\mathrm{Zn}$ atom shows a perpendicular displacement with respect to the porphyrin plane by $0.47 \AA$ due to the interaction with the $\mathrm{NO}_{2}$ group. This central atom displacement exhibited a square-pyramidal coordination state which has been observed in metalloporphyrins attached to five-coordinated species such as zinc, magnesium, cobalt(II) and iron [24].

The interaction among two porphyrins and one $\mathrm{NO}_{2}$ molecule provided a great amount of information regarding not only the configuration of the molecules in a stacking-like arrangement, but also the disruption of this configuration when a gas molecule was introduced. The ground energy configuration of

(a)

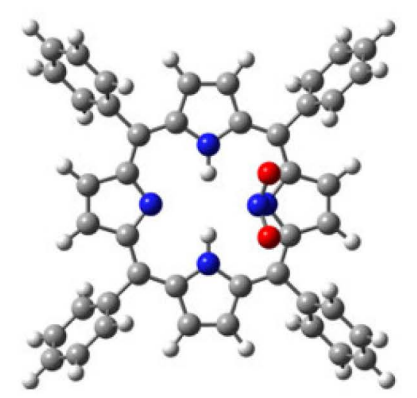

(c)

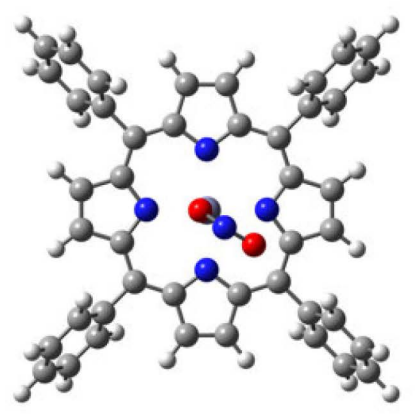

(b)

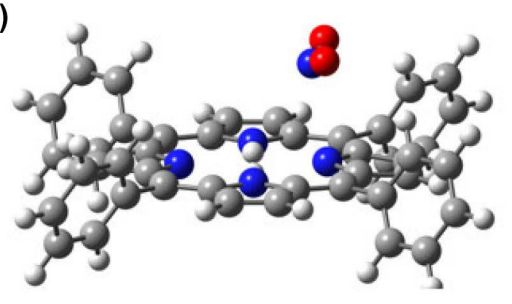

(d)

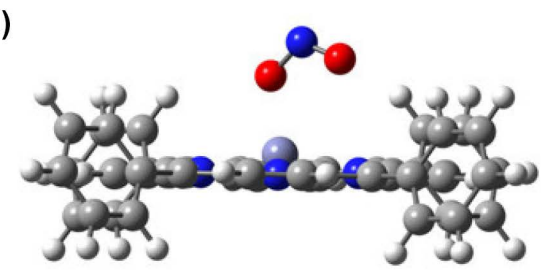

Figure 8. (a,b) $\mathrm{H}_{2} \mathrm{TPP}$ and (c,d) ZnTPP molecules interacting with $\mathrm{NO}_{2}$. 
two metal free and zinc molecular systems was optimized previous to the gas insertion. For the metal free system, the two molecules exhibited a semi parallel arrangement, where the shortest perpendicular distance between both molecules was $4.38 \AA$. In addition, the system showed a shift between molecular cores of $6.82 \AA$ (center-center distance) in addition to an in-plane rotation of $33.8^{\circ}$ due to the presence of the external benzene groups. One of the molecules exhibited a planar configuration similar to the single metal free molecule, but the other, exhibited a slightly out-of-plane core saddle-shaped distortion, which can be attributed to the tetraphenyl porphyrin ring interacting with one of the external phenyl groups of the other molecule. This arrangement showed changes in the dihedral angles between the mean plane of the porphyrins and the peripheral phenyl groups that ranged between $61.28^{\circ}$ to $90^{\circ}$, being the perpendicular orientation of one of the phenyl groups on top of the other molecule [24] (images not shown here). For the next step, different initial configurations were explore with the nitrogen dioxide group in between the porphyrin molecules. The lowest energy configuration showed that the separation between metal free molecules increased to $9.25 \AA$ (center-center distance). In addition, one of the porphyrin molecules showed an important deviation from the parallel plane of $59.79^{\circ}$, with one of its external phenyl groups pointing towards the other molecular core. The nitrogen dioxide molecule lied in between the porphyrin groups with its molecular plane slightly tilted towards one of the porphyrin central ring. From this result, it is clear that the molecular alignment is definitely disrupted by the presence of the gas. The ground state energy for this arrangement was $-20.36 \mathrm{~kJ} / \mathrm{mol}$. The frontal and lateral geometries for the metal free system can be seen in Figure 9(a) and Figure 9(b).

On the other hand, the lowest energy configuration of two Zinc tetraporphyrin molecules showed a columnar-like arrangement, where one of the Zinc centers is slightly shifted from the vertical alignment. The distance between Zinc-Zinc atoms was $6.08 \AA$, whereas the horizontal shifting distance between Zinc-Zinc atoms was $3.13 \AA$.

In this arrangement, both molecules show planar configurations with the Zinc atoms perfectly aligned within the ring planes. In addition, the dihedral angles of the external phenyl groups did not show any change from the ground state molecular conformation. This two-molecular configuration was used as a initial arrangement for the gas interaction. The nitrogen dioxide molecule was introduced at different position in between the porphyrins molecules and the lowest energy configuration is shown in Figure 9(c), Figure 9(d). The molecular ring separation increased to $6.47 \AA$, while the Zinc-Zinc distance decreased to $5.09 \AA$. This difference is related to the out-of-plane displacement of the Zinc atoms due to the $\mathrm{NO}_{2}$ interaction, which also induced a small molecular distortion for both porphyrin cores. In addition, the Zinc-Zinc distance decreased by $0.60 \AA$ from the original position. The in-plane molecular rotation between the two ZnTPP molecules also changed $26.87^{\circ}$ showing a better perpendicular alignment between both zinc porphyrin molecules. The nitrogen dioxide molecule remained 
(a)

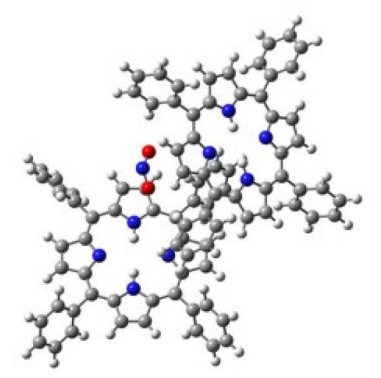

(c)

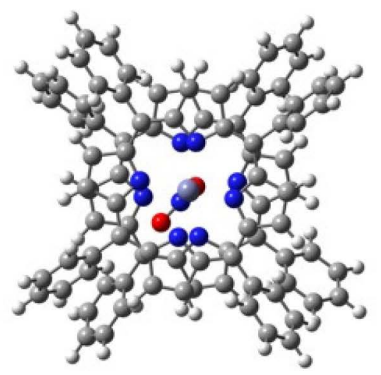

(b)

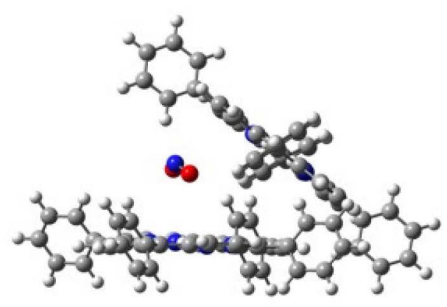

(d)

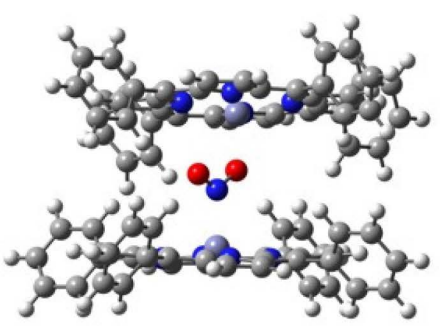

Figure 9. Two (a) (b) $\mathrm{H}_{2} \mathrm{TPP}$ and (c) (d) ZnTPP molecules interacting with a $\mathrm{NO}_{2}$ molecule.

in between the two porphyrins cores in a $N$-nitro configuration towards one of the porphyring rings. The nitrogen atom pointed towards one of the zinc atoms at a distance of $2.16 \AA$, while one of the oxygens point towards the opposite zinc atom with a separation of $2.10 \AA$. The $\angle \mathrm{O}-\mathrm{N}-\mathrm{O}$ was $119.02^{\circ}$. The binding energy for this arrangement was $-18.50 \mathrm{~kJ} / \mathrm{mol}$.

In a different simulation, not shown here, two $\mathrm{NO}_{2}$ molecules were introduce between the two ZnTPP molecules and each coordinate to a different central metal atom. The two nitrogen dioxide groups remained closed, and the distance and alignment between the porphyrin molecules were hardly disrupted, suggesting an preferent coordination between the gas and the porphyrin metal centers.

Finally, preliminary results for the UV-Vis calculated spectra for the $\mathrm{H}_{2}$ TPP molecule showed that the small bathochromic shift of the Soret peak after gas interaction is related to the distortion of the electronic structure of the macrocycle ring due to the ring deformation, whereas the appearance of the $670 \mathrm{~nm}$ peak is due to the absorption of the $\mathrm{NO}_{2}$ species to meso-carbon positions. As the $\mathrm{NO}_{2}$ concentration increased, more molecules coordinate to meso-carbon atoms increasing the peak size as observed in the experimental UV-Vis spectra. On the other hand, for the $\mathrm{Zn}$ metal porphyrin system, the large bathochromic shif of the Soret peak is due to the coordination of $\mathrm{NO}_{2}$ groups to the zinc metal center. In addition, we found distinct peaks between $520 \mathrm{~nm}$ and $540 \mathrm{~nm}$, which are very close to the $521 \mathrm{~nm}$ experimental value.

\section{Discussion}

Although previous results suggested that porphyrin films exposed to $\mathrm{NO}_{2}$ can 
recover over time or by exposing the films to elevated temperatures [10], from microscopy images, we showed important differences in the film integrity after gas exposure. For the metal-free system, our results indicate a strong degradation of the film caused by the interaction of $\mathrm{NO}_{2}$ groups with the macrocycle ring resulting in a progressive irreversible damage of the film interface. In addition, from the simulations of the two porphyring systems, the $\mathrm{H}_{2}$ TPP molecules exhibited very little distortion of their individual macrocycle rings, but an important collaps in the packing arrangement which also contributes to the irreversible damage of the film. Other groups suggested that the irreversible damage of a cobalt tetraphenyl porphyrin film in the presence of $\mathrm{NO}_{2}$ could be attributed to an irreversible chemical transformation due the formation of dications and the nitration on the porphyrin ring [25].

On the other hand, the film with the molecules coordinated to the zinc central atom showed the progressive absorption of $\mathrm{NO}_{2}$ gas in between the different layers of the molecular film, which in addition, provided a better stability of the molecular arrangement. From SEM and AFM results, we noticed that the film interface remained undamaged, although a swelling of the film is observed in agreement with DFT simulations.

When analyzing the absorption spectra of the $\mathrm{H}_{2}$ TPP and ZnTPP films after gas exposure, bathochromic shifts of the Soret peaks were observed in both cases, although the largest difference was obtained in the later case. From the DFT simulations, the arrangement with the $\mathrm{H}_{2} \mathrm{TPP}$ molecules exhibited very little distortion of the individual macrocycle rings. In contrast, the presence of the gas in between the two $\mathrm{Zn}$ porphyrin molecules, not only modified the macrocycles and $\mathrm{Zn}$ atoms distances, but also distorted the planarity of both molecules affecting the $\pi$ conjugation system contributing to the large red shift. On the other hand, it has been observed that the presence of $\mathrm{NO}_{2}$ either as gas or in substituted porphyrins molecules show the presence of peaks above the $600 \mathrm{~nm}$ wavelength, which is consistent with our results. Furthermore, as the gas concentration increased in our experiments, the size of the long wavelength peaks increased too, which reflects the amount of gas in the system. From our findings, shorter peaks were observed for the surface gas interaction, while larger peaks were seen for the system with gas trapped within the film.

The in-plane conductivity results showed a larger voltage increase for the metal free system and we noticed a constant saturation voltage over the time of the experiment regardless of the surface degradation due to the thickness of the films. Finally, the contact angle plots showed a decrease in the hydrophobic nature of the interface after gas exposure due to competitive changes in roughness and surface chemical transformation.

\section{Conclusions}

In this work, metal-free and zinc tetraphenyl porphyrin films were employed as gas $\mathrm{NO}_{2}$ gas sensors. In both cases, an irreversible degradation of the films was 
observed, which was most important for the metal free system due to the attack of the porphyrin ring by $\mathrm{NO}_{2}$ species. For the metal porphyrin film, the metal mediated the absorption of the gas in between the film layers by trapping $\mathrm{NO}_{2}$ gas molecules inside the molecular arrangement. These results were consistent with experimental UV-Vis, SEM, AFM and conductivity results. By using DFT and TD-DFT computer simulations, we were able to validate the experimental findings for the gas interaction mechanisms for each system. Finally, porphyrin films are sensitivity materials for the detection of $\mathrm{NO}_{2}$ and by using evaporation techniques, it is possible to select the mechanism for gas interaction as well as the detection time and life span of the sensor by modifying the presence of the central metal atom and the thickness of the molecular film.

\section{Acknowledgements}

MR gratefully acknowledges DGAPA-PAPIIT project number IN108017 and DGTIC-UNAM project number LANCAD-UNAM-DGTIC-289. JM Rivera acknowledges scholarship from CONACYT-Mexico. MR and YA Wang acknowledge Westgrid, Canada. Authors acknowledge Dr. Carlos Magana and Samuel Tehuacanero C. for SEM technical assistance and Mr. Arturo Martinez for technical support.

\section{Conflicts of Interest}

The authors declare no conflicts of interest regarding the publication of this paper.

\section{References}

[1] Szulczynski, B. and Gebicki, J. (2017) Currently Commercially Available Chemical Sensors Employed for Detection of Volatile Organic Compounds in Outdoor and Indoor Air. Environments, 4, 1-15.

[2] Wang, B., Zuo, X., Wu, Y., Chen, Z., He, C. and Duan, W. (2011) Comparative Gas Sensing in Copper Porphyrin and Copper Phthalocyanine Spin-Coating Films. Sensors and Actuators B: Chemical, 152, 191-195. https://doi.org/10.1016/j.snb.2010.12.006

[3] Zhou, R., Josse, F., Göpel, W., Öztürk, Z.Z. and Bekaroğlu, Ö (1996) Phthalocyanines as Sensitive Materials for Chemical Sensors. Applied Organometallic Chemistry, 10, 557-577. https://doi.org/10.1002/(SICI)1099-0739(199610)10:8<557::AID-AOC521>3.0.CO;2 $\underline{-3}$

[4] Bohrer, F.I., Sharoni A., Colesniuc, C., Park, J., Schuller, I.K., Kummel, A.C. and Trogler, W.C. (2007) Gas Sensing Mechanism in Chemiresistive Cobalt and Metal-Free Phthalocyanine Thin Films. Journal of the American Chemical Society, 129, 5640-5646. https://doi.org/10.1021/ja0689379

[5] Bajju, G.D., Kundan, S., Bhagat, M., Gupta, D., Kapahi, A. and Devi, G. (2014) Synthesis and Spectroscopic and Biological Activities of $\mathrm{Zn}$ (II) Porphyrin with Oxygen Donors. Bioinorganic Chemistry and Applications, 2014, 782762.

[6] Dunbar, A.D.F., Brittle, S., Richardson, T.H., Hutchinson, J. and Hunter, C.A. 
(2010) Detection of Volatile Organic Compounds Using Porphyrin Derivatives. The Journal of Physical Chemistry B, 114, 11697-11702. https://doi.org/10.1021/jp102755h

[7] Tredgold, R.H., Young, M.C.J., Hodge, P. and Hoorfar, A. (1985) Gas Sensors Made From Langmuir-Blodgett Films of Porphyrins. IEEE Proceedings, 132, 151-156.

[8] Farias de Sales, N. and Mansur, H.S. (2008) Chemsensor of $\mathrm{NO}_{2}$ Gas Based on Porphyrin of 5,10,15,20-Tetraphenylporphyrin LB and LS Films. Materials Research, 4, 477-482. https://doi.org/10.1590/S1516-14392008000400017

[9] Evyapan, M. and Dunbar, A.D.F. (2015) Improving the Selectivity of a Free Base Tetraphenylporphyrin Based Gas Sensor for $\mathrm{NO}_{2}$ and Carboxylic Acid Vapors. Sensors and Actuators B: Chemical, 206, 74-83.

[10] Richardson, T.H., Dooling, C.M., Jones, L.T. and Brook, R.A. (2005) Development and Optimization of Porphyrin Gas Sensing LB Films. Advances in Colloid and Interface Science, 116, 81-96.

[11] Uttiya, S., Pratontep, S., Bhanthumnavin, W., Buntem, R. and Kerdcharoen, T. (2008) Volatile Organic Compounds Sensor Array Based on Zinc Phthalocyanine and Zinc Porphyrin Thin Films. 2nd IEEE International Conference: Nanoelectronics Conference, 1, 618-623.

[12] Roales, J., Pedrosa, J.M., Castillero, P., Cano, M., Richardson, T.H., Barranco, A. and Gonzalez-Elipe, A.R. (2012) Selective Detection of Volatile Organic Compounds by Spectral Imaging of Porphyrin Derivatives Bound to $\mathrm{TiO}_{2}$ Porous Films ACS Applied Materials \& Interfaces, 4, 5147-5154. https://doi.org/10.1021/am3010169

[13] Gouterman, M. (1961) Spectra of Porphyrins. Journal of Molecular Spectroscopy, 6, 138-163. https://doi.org/10.1016/0022-2852(61)90236-3

[14] van Faassen, E.E., Bahrami, S., Feelisch M., Hogg, N., Kelm, M., Kim-Shapiro, D.B., Kozlov, A.V., Li, H., Lundberg, Jo. O., Mason, R., Nohl, H., Rassaf, T., Samouilov, A., Slama-Schwok, A., Shiva, S., Vanin, A.F., Weitzberg, E., Zweier, J. and Gladwin, M.T. (2009) Nitrite as Regulator of Hypoxic Signaling in Mammalian Physiology. Medicinal Research Reviews, 29, 683-741. https://doi.org/10.1002/med.20151

[15] Zhang, T.T., Liu, Y.D. and Zhong, R.G. (2015) Iron(II) Porphyrins Induced Conversion of Nitrite into Nitric Oxide. A Computational Study. Journal of Inorganic Biochemistry, 150, 126-132. https://doi.org/10.1016/j.jinorgbio.2015.06.005

[16] Turias, F., Sola, M., Falivene, L., Cavallo L. and Poater, A. (2016) Iron(II) Porphyrins Induced Conversion of Nitrite into Nitric Oxide. A Computational Study. Structural Chemistry, 27, 409-417. https://doi.org/10.1007/s11224-015-0670-0

[17] Kalimuthu, P., Sivanesan, A. and Abraham John, S. (2012) Fabrication of Optochemical and electrochemical sensors using Thin Films of Porphyrin and Phthalocyanine Derivatives. Journal of Chemical Sciences, 124, 1315-1325.

https://www.ias.ac.in/article/fulltext/jcsc/124/06/1315-1325 https://doi.org/10.1007/s12039-012-0330-5

[18] Muthukumar, P. and Abraham John, S. (2011) Highly Sensitive Detection of HCl Gas Using a Thin Film of Meso-Tetra(4-pyridyl)porphyrin Coated Glass Slide by Optochemical Method. Sensors and Actuators B: Chemical, 159, 238-244. https://doi.org/10.1016/j.snb.2011.06.079

[19] Giovannetti, R. (2012) The Use of Spectrophotometry UV-Vis for the Study of Porphyrins. In: Uddin, J., Ed., Macro to Nano Spectroscopy, InTech, Rijeka, 87-108.

[20] Sun, Z.-C., She, Y.-B., Zhou, Y., Song, X.-F. and Li, K. (2016) Synthesis, Characterization and Spectral Properties of Substituted Tetraphenylporphyrin Iron Chloride 
Complexes. Molecules, 16, 2960-2970.

[21] Kurtikyan, T.S., Hovhannisyan, A.A., Iretskii, A.V. and Ford, P.C. (2009) Six-Coordinate Nitro Complexes of Iron(III) Porphyrins with trans S-Donor Ligands. Oxo-Transfer Reactivity in the Solid State. Inorganic Chemistry, 48, 11236-11241. https://doi.org/10.1021/ic901722g

[22] Li, J.P., Tredgold, R.H., Jones, R. and Hodge, P. (1990) Interactions of Nitrogen Dioxide with Langmuir-Blodgett Films of a Copper Porphyrin. Thin Solid Films, 186, $167-176$.

[23] Aydin, M. (2013) DFT and Raman Spectroscopy of Porphyrin Derivatives: Tetraphenylporphine (TPP). Vibrational Spectroscopy, 68, 141-152.

https://doi.org/10.1016/j.vibspec.2013.06.005

[24] Scheidt, W.R. (2000) 16/Systematics of the Stereochemistry of Porphyrins and Metalloporphyrins. In: Kadish, K.M., Smith, K.M. and Guilard, R., Eds., The Porphyrin Handbook Vol. 3/Inorganic, Organometallic and Coordination Chemistry, Academic Press, San Diego, 55.

[25] Kurtikyan, T.S. and Stepanyan, T.G. (1998) Oxidation Sequence of Sublimed Meso-Tetraphenylporphyrinatocobalt(II) Films in an Atmosphere of Nitrogen Dioxide. Russian Chemical Bulletin, 47, 695-698. 